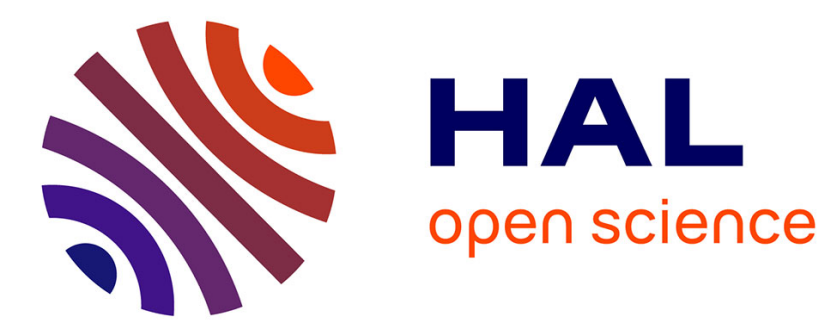

\title{
Effect of the curvature and the $\beta$ parameter on the nonlinear dynamics of a drift tearing magnetic island
}

Magali Muraglia, Olivier Agullo, Masatoshi Yagi, Sadruddin Benkadda, Beyer

Peter, Xavier Garbet, Sanae -I. Itoh, Kimitaka Itoh, Abhijit Sen

\section{- To cite this version:}

Magali Muraglia, Olivier Agullo, Masatoshi Yagi, Sadruddin Benkadda, Beyer Peter, et al.. Effect of the curvature and the $\beta$ parameter on the nonlinear dynamics of a drift tearing magnetic island. Nuclear Fusion, 2009, 49, pp.055016. hal-00578079

\section{HAL Id: hal-00578079 \\ https://hal.science/hal-00578079}

Submitted on 18 Mar 2011

HAL is a multi-disciplinary open access archive for the deposit and dissemination of scientific research documents, whether they are published or not. The documents may come from teaching and research institutions in France or abroad, or from public or private research centers.
L'archive ouverte pluridisciplinaire HAL, est destinée au dépôt et à la diffusion de documents scientifiques de niveau recherche, publiés ou non, émanant des établissements d'enseignement et de recherche français ou étrangers, des laboratoires publics ou privés. 


\title{
Effect of the curvature and the $\beta$ parameter on the nonlinear dynamics of a drift tearing magnetic island
}

\author{
M. Muragliaa ${ }^{1,2,3}$, O. Agullo ${ }^{1,2}$, M. Yagi ${ }^{1,4}$, S. Benkadda ${ }^{1,2}$,

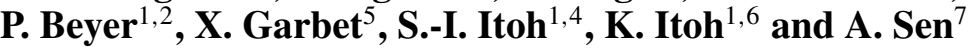 \\ ${ }^{1}$ France-Japan Magnetic Fusion Laboratory, LIA 336 CNRS \\ 2 PIIM Laboratory, UMR 6633 CNRS-University of Provence, Marseille \\ ${ }^{3}$ Interdisciplinary Graduate School of Engineering Sciences, Kyushu University \\ ${ }^{4}$ Research Institute for Applied Mechanics, Kyushu University, Kasuga 816-8580, Japan \\ ${ }^{5}$ CEA, IRFM, 13108, St-Paul-Lez-Durance, France \\ ${ }^{6}$ National Institute for Fusion Science, Toki, 509-5292, Japan \\ ${ }^{7}$ Institute for Plasma Research, Bhat, Gandhinagar 382428, India
}

Received 20 December 2008, accepted for publication 18 March 2009

Published 28 April 2009

Online at stacks.iop.org/NF/49/055016

\begin{abstract}
We present numerical simulation studies of 2D reduced MHD equations investigating the impact of the electronic $\beta$ parameter and of curvature effects on the nonlinear evolution of drift tearing islands. We observe a bifurcation phenomenon that leads to an amplification of the pressure energy, the generation of $E \times B$ poloidal flow and a nonlinear diamagnetic drift that affects the rotation of the magnetic island. These dynamical modifications arise due to quasilinear effects that generate a zonal flow at the onset point of the bifurcation. Our simulations show that the transition point is influenced by the $\beta$ parameter such that the pressure gradient through a curvature effect strongly stabilizes the transition. Regarding the modified rotation of the island, a model for the frequency is derived in order to study its origin and the effect of the $\beta$ parameter. It appears that after the transition, an $E \times B$ poloidal flow as well as a nonlinear diamagnetic drift are generated due to an amplification of the stresses by pressure effects.
\end{abstract}

PACS numbers: 52.30.Cv, 52.35.Py, 52.35.Ra, 52.55.Fa

(Some figures in this article are in colour only in the electronic version)

\section{Introduction}

In tokamak and space plasmas, confinement can be affected by instabilities and in particular, at resonant surfaces, magnetohydrodynamics activity can lead to the generation of magnetic islands reaching a macroscopic width. Solar flares [1], energy release events in the geotail [2] or tokamak internal disruptions, also known as sawtooth oscillations, are linked to such reconnection phenomena. Diamagnetic effects and self-generated zonal flows can modify the saturated island width via bifurcation mechanisms [3]. The rotation frequency of the island can also be nonlinearly affected with a strong dependence on the transport coefficients and on the competition between the Reynolds and Maxwell stresses [4]. This can have a significant physical consequence, for example in a tokamak, where such a nonlinear effect on the rotation can lead to a slowing down of the plasma through locking to the resistive wall producing in turn a degradation of the plasma and/or triggering a transport barrier [5]. Likewise curvature effects can also modify the nature of island dynamics. Magnetic islands can in particular coexist with pressure driven instabilities such as interchange modes and/or turbulence. Several experiments report the coexistence of turbulence and MHD activities showing some correlated effects $[6,7]$. Numerical studies of the interaction between double tearing modes and micro-turbulence to delineate the interaction between zonal flows and the latter in the growing phase of the double tearing instability have also been performed in [8]. More recently, in [9] an investigation of the interaction of a 2D electrostatic turbulence with an island whose dynamics is governed by a generalized Rutherford equation has been carried out. However the study neglects the potentially stabilizing influence of the magnetic structure on the turbulence thereby precluding any multi-scale interaction between MHD and turbulence. In this paper we study the dynamics of a magnetic island in the presence of interchange effects but limit ourselves to the situation where the interchange modes in the system are linearly stable. We find that the whole 
system does not generate turbulence in the nonlinear stage but exhibits a complex dynamics arising mainly due to quasilinear effects. Our investigations are based on linear and nonlinear simulations of a set of reduced fluid equations (a three field model) through which we examine the origin and the influence of zonal flows on the magnetic island dynamics in the presence of interchange effects. The magnitude of the pressure gradient appears to be a key parameter of the dynamics controlling both the generation of the zonal flow and the development of a nonlinear transition in the system.

The paper is organized as follows. In section 2, the model equations are introduced. In section 3, a linear analysis of the model is done in order to understand the role of the equilibrium magnetic field in the stabilization of the electromagnetic interchange modes. In section 4 , the description and the analysis of the dynamics are done. In section 5, the origin of the island poloidal rotation is investigated. Section 6 presents a summary and conclusions of the paper.

\section{Model system}

Our model system is a three fields model corresponding to a reduced magnetohydrodynamic description of the fluid equations [10] and which provides a minimal framework for including both the interchange and the tearing mode phenomena in a plasma. The model consists of a set of three coupled equations for the electrostatic potential $\phi$, the pressure of the electron $p$ and the magnetic flux $\psi$. We suppose that the magnetic field is dominated by a constant component $B_{0 z}$ along the $z$-direction. The time evolution of the three fields are described by

$$
\begin{aligned}
& \partial_{t} \nabla_{\perp}^{2} \phi+\left[\phi, \nabla_{\perp}^{2} \phi\right]=\left[\psi, \nabla_{\perp}^{2} \psi\right]-\kappa_{1} \partial_{y} p+v \nabla_{\perp}^{4} \phi, \\
& \partial_{t} p+[\phi, p]=-v_{\star}\left(\left(1-\kappa_{2}\right) \partial_{y} \phi+\kappa_{2} \partial_{y} p\right) \\
& \quad+C^{2}\left[\psi, \nabla_{\perp}^{2} \psi\right]+\chi_{\perp} \nabla_{\perp}^{2} p, \\
& \partial_{t} \psi+[\phi-p, \psi]=-v_{\star} \partial_{y} \psi+\eta \nabla_{\perp}^{2} \psi,
\end{aligned}
$$

where $v_{\star}=\frac{\beta L_{\perp}}{2 \Omega_{\mathrm{i}} \tau_{\mathrm{A}} L_{\mathrm{p}}}$. The sum of the electron and ion momentum evolution equations leads to the plasma equation of motion, equation (1), where $v$ is the viscosity. Equation (2) comes from the energy conservation equation where $\chi_{\perp}$ is the diffusivity. Equation (3) is Ohm's law (electron parallel momentum equation) with $\eta$ being the resistivity. $\beta=\frac{p_{0}}{B_{0 z}^{2} / 2 \mu_{0}}$ is the ratio of the electron thermal energy to the magnetic energy ( $p_{0}$ being the amplitude of the equilibrium pressure), $L_{\mathrm{p}}$ is the pressure gradient length, $L_{\perp}$ is a magnetic shear length, $R_{0}$ is the major plasma radius, $\Omega_{\mathrm{i}}=\frac{e B_{0 z}}{m_{\mathrm{i}}}$ is the ion cyclotron frequency and $\tau_{\mathrm{a}}$ is the Alfvèn time. Equations (1)-(3) are normalized as follows:

$$
\begin{gathered}
\frac{t}{\tau_{\mathrm{A}}} \rightarrow t, \quad \frac{x}{L_{\perp}} \rightarrow x, \\
\frac{\psi}{L_{\perp} B_{0 z}} \rightarrow \psi, \quad \frac{\phi}{L_{\perp} v_{\mathrm{A}} B_{0 z}} \rightarrow \phi, \quad \frac{L_{\mathrm{p}}}{L_{\perp} p_{0}} p \rightarrow p,
\end{gathered}
$$

where $v_{\mathrm{A}}=B_{0 z} / \mu_{0} n m_{\mathrm{i}}=L_{\perp} / \tau_{\mathrm{a}}$ is the characteristic Alfvèn speed. $\kappa_{\mathrm{i}}$ parameters are linked to the curvature and to the pressure gradient $\left(\kappa_{1}=2 \Omega_{\mathrm{i}} \tau_{\mathrm{A}} \frac{L_{\perp}}{R_{0}}\right.$ and $\left.\kappa_{2}=\frac{10 L_{\mathrm{p}}}{3 R_{0}}\right)$, so these
Table 1. Effect of the $\beta$ parameter on $v^{\star}$ and $C$.

\begin{tabular}{lll}
\hline$\beta$ & $v^{\star}$ & $C^{2}$ \\
\hline 0.001 & $2 \times 10^{-3}$ & $3.3 \times 10^{-3}$ \\
0.005 & $10^{-2}$ & $1.67 \times 10^{-2}$ \\
0.015 & $3 \times 10^{-2}$ & $5 \times 10^{-2}$ \\
0.025 & $5 \times 10^{-2}$ & $8.33 \times 10^{-2}$ \\
\hline
\end{tabular}

parameters control the interchange instability. On the other hand, in equation (2), the tearing mode dynamics is controlled by the coupling parameter $C^{2}=\frac{5 \beta}{6 \Omega_{\mathrm{i}}^{2} \tau_{\mathrm{A}}^{2}}$. More precisely, this parameter controls the coupling between pressure and the magnetic flux. The nature of the linear and nonlinear dynamics of the magnetic island depends strongly on the strength of the coupling. For a high $\beta$ plasma, since the coupling is strong, the pressure and the magnetic flux control the island dynamics, whereas for a low $\beta$ plasma, the island dynamics is governed by the interaction between the flow and the magnetic flux. In our model we assume the electron temperature to be constant and the ions to be cold. The cold ion limit is physically realistic since the ion temperature does not significantly affect the stability of the tearing mode. As a further simplification we have also neglected the parallel ion dynamics in the energy balance equation (equation (2)). Equations (1)-(3) are solved numerically using a finite difference scheme in the $x$ direction, including an Arakawa algorithm [11] for an accurate conservation of the Poisson brackets [., .] and a pseudo-spectral method in the $y$ direction, including an appropriate de-aliasing scheme.

\section{Nature of the tearing modes and the influence of the curvature parameter $\kappa_{1}$}

We now study the influence of the interchange mechanism on the magnetic reconnection when the gradient scale length of the pressure, $L_{\mathrm{p}}$, is of the order of the size of the island: we set $L_{\mathrm{p}}=L_{\perp}$. We are interested in large islands, i.e. islands with widths $w$ such that $a \gtrsim w \gg \rho_{\mathrm{s}}$ where $a$ is the minor radius and $\rho_{\mathrm{s}}$ is the hybrid Larmor radius $\left(\rho_{\mathrm{s}}=c_{\mathrm{S}} / \Omega_{\mathrm{i}}\right.$, where $c_{\mathrm{s}}$ is the ion sound velocity), and we have chosen $L_{\perp}=0.24 \mathrm{~m}$. The numerical values of other parameters are taken to be $R_{0}=2.24 \mathrm{~m}$ and $\Omega_{\mathrm{i}} \tau_{\mathrm{A}}=0.5$. These numerical values are typical of the TORE SUPRA device for an island width of about $1 / 3$ the minor radius and lead to $\kappa_{1} \sim 0.11$ and $\kappa_{2} \sim 0.36$. The widths of the numerical integration box are set to $L_{x}=2 \pi L_{\perp}$ and $L_{y}=5 \pi L_{\perp}$. The values of the coefficients $C(\beta)$ and $\omega_{\star}(\beta)=k_{y} v_{\star}$ are determined for four different values of $\beta$ in the range of $10^{-3}$ to $2.5 \times 10^{-2}$ (see table 1 ). Transport coefficients $(v, \eta, \chi)$ are all set to $10^{-4}$ which correspond to renormalized coefficients to include effects of microscopic turbulence [12] . The equilibrium magnetic field $\boldsymbol{B}_{0 y}=B_{0} \hat{\boldsymbol{y}}$, based on the Harris current sheet model [13], is chosen to be of the form,

$$
B_{0}(x)=\tanh \left(\frac{x-L_{x} / 2}{a_{t}}\right) .
$$

The parameter $a_{t}=0.75$ controls the width of the profile, $\psi_{0}^{\prime}(x)=B_{0}(x)$. With such a profile, the parameter $\Delta^{\prime}$ (the tearing mode stability index) can be explicitly computed taking 

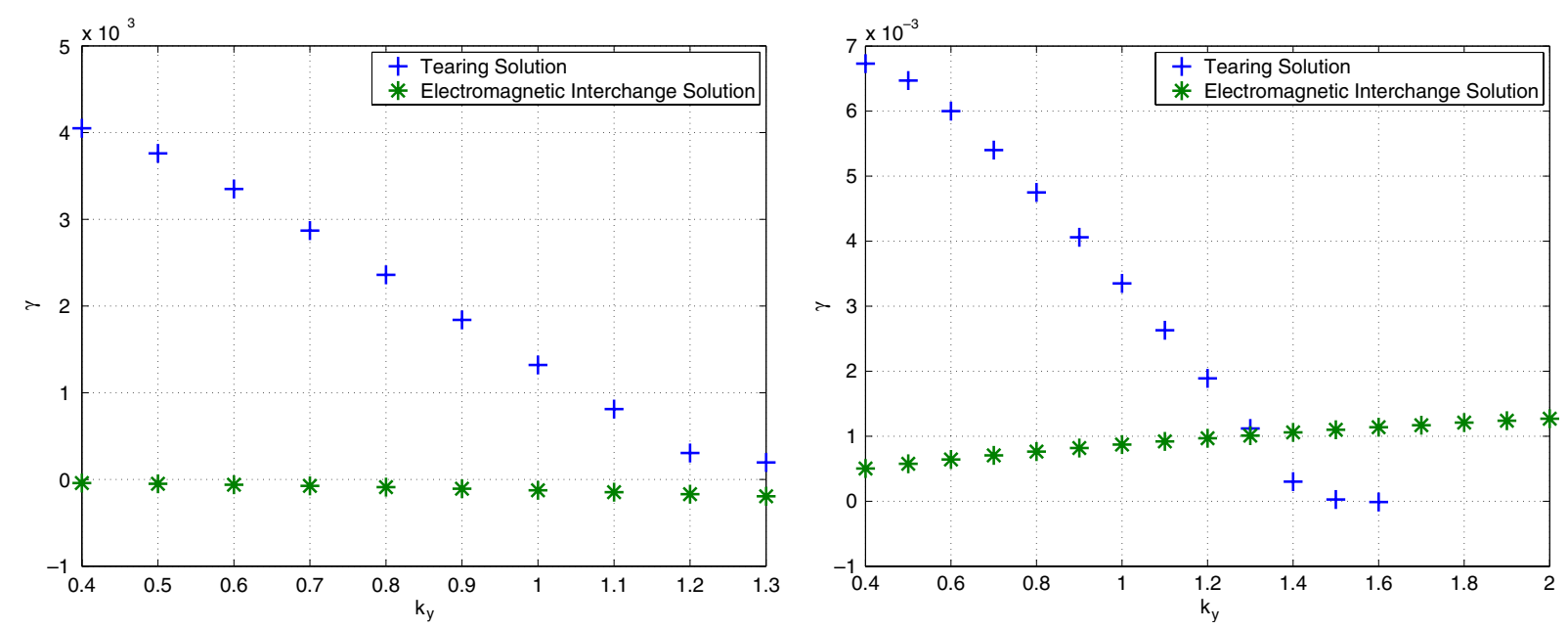

Figure 1. Linear growth rate $\gamma\left[\tau_{\mathrm{A}}^{-1}\right]$ versus poloidal mode number $k_{y}$ for a simulation with $\beta=10^{-3}$. (Left) $v^{\star}=2 \times 10^{-3}$, $C=3.33 \times 10^{-3}, \kappa_{1}=0.1071, \kappa_{2}=0.3571$ and $\mu=\chi_{\perp}=\eta=10^{-4}$. (Right) $v^{\star}=10^{-2}, C=2 \times 10^{-3}, \kappa_{1}=5, \kappa_{2}=0.3571$, $\mu=\chi_{\perp}=10^{-5}$ and $\eta=10^{-4}$.

into account the boundary conditions, for modes evolving slowly on the Alfvén time scale and further neglecting the viscous and interchange corrections. Introducing $\hat{k}=a_{t} k_{y}$, we have

$$
a_{t} \Delta^{\prime}=2(1 / \hat{k}-\hat{k})+\hat{\Delta}_{\mathrm{b} \cdot \mathrm{c}}
$$

where

$$
2 \hat{\Delta}_{\mathrm{b} \cdot \mathrm{c}}^{-1}=-\int_{0}^{L_{x} /\left(2 a_{t}\right)} \mathrm{d} y \exp (2 \hat{k} y) /(1+\tanh (y) / \hat{k})^{2}
$$

is a correction linked to the finite radial distance of the walls.

Let us investigate the stability of the modes modelled by equations (1)-(3) with the given numerical values of the parameters in the presence of such an equilibrium. Figure 1, shows the growth rate of the electromagnetic interchange and the tearing branches, as functions of the poloidal wave number, for the parameter values given above. The left graph has been obtained using the parameters chosen in this work with $\beta=0.001$. Tearing instability has the largest growth rate at $k_{y}^{\text {tear }}=2 \pi / L Y=0.4$, for which $\Delta^{\prime}=6$ and $\gamma_{\text {tear }} \sim 0.0042$. This is clearly smaller than the one we would obtain in the classical tearing limit, i.e. if we set all the parameters to zero except $\eta\left(\gamma_{\text {tear class }}=0.0072\right)$. It is instructive to note also that in this parametric regime the interchange branch is stable for any wave number. From an electrostatic point of view, with such parameters, interchange would have been unstable for $k_{y}<8$ and would have given a scale separation between both instabilities $\left(k_{y}^{\text {int elec }} / k_{y}^{\text {tear }} \sim 7, \gamma^{\text {int elec }} / \gamma^{\text {tear }} \ll 1\right)$. Let us focus on the tearing branch. Linearization of equations (1)-(3) in the vicinity of the resonance shows that curvature effects weakly modify the growth rate if $\kappa_{1} v_{\star}\left(k_{y} / k_{x}\right)^{2} / \gamma^{2} \ll 1$ and $\kappa_{2} \ll 1$, which is true in our case. Considering the fact that the linear regime is also not controlled by viscous phenomena [14], it follows that, linearly, this system develops approximately drift tearing modes. The actual nature of these modes is controlled mainly by the ratio $P / P_{\text {cr }}$ and $\gamma / \omega_{\star}$, where $P=$ $v / \eta=1$ is the Prandtl number and $P_{\mathrm{cr}}=\left(\Delta^{\prime}\left(\eta / k_{y}\right)^{1 / 3}\right)^{6 / 5}$. In our cases, for any $\beta$, the first ratio is always smaller than 1 . It implies that when $\gamma / \omega_{\star}>1$, one gets the visco-tearing regime with a growth rate scaling law $\gamma_{\mathrm{vt}} \sim 0.47 \Delta^{\prime} \eta^{2 / 3} P^{-1 / 6} k_{y}^{1 / 3}$.

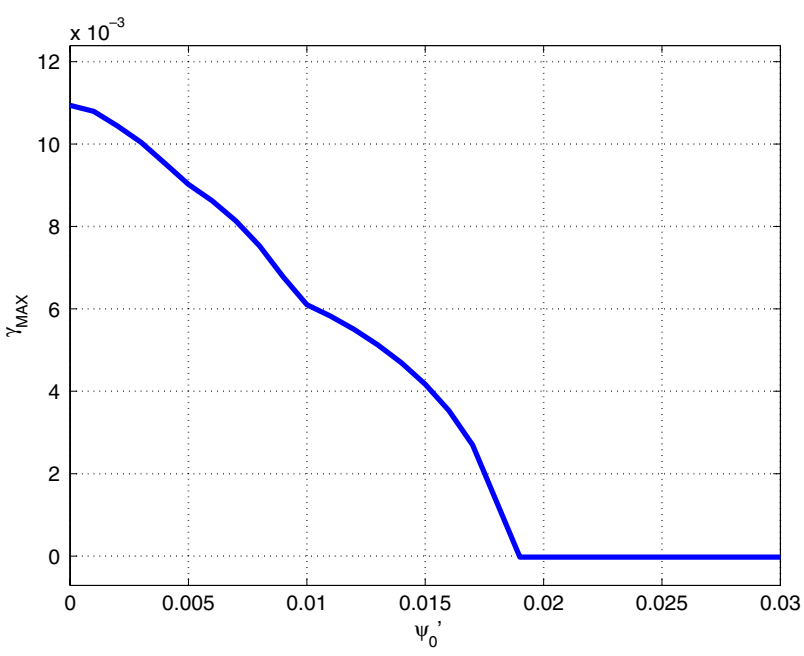

Figure 2. Stability of electromagnetic interchange modes versus $\psi_{0}^{\prime}$, far from the resonant surface. Same parameter values as figure 1 .

When $\gamma / \omega_{\star}<1$, we recover the visco-drift tearing regime with the growth rate $\gamma_{\mathrm{vdt}}$ [15]. For instance, for $\beta=0.001$ and $k_{y}=0.4$, we have $\gamma_{\mathrm{vt}} / \omega_{\star} \sim 5.5>1$ and $\gamma=0.0042 \sim$ $\gamma_{\mathrm{vt}}=0.0045$. For $k_{y}=1.2$, we have $\gamma_{\mathrm{vt}} / \omega_{\star} \sim 0.2<1$ and $\gamma=0.00035 \sim \gamma_{\mathrm{vdt}}=0.0004$.

The right graph of figure 1 shows that there exist regimes where the interchange branch is unstable and has the largest growth rate at small scales. The study of such regimes is out of the scope of this paper. We also remark that the instability does not necessarily develop in the vicinity of a resonant surface, but in that case, the effect of the magnetic field on the stability of interchange like modes can be investigated by setting $\psi_{0}^{\prime}=B_{0}=$ Cte and using some Fourier analysis. Figure 2 shows the linear growth rate of interchange modes versus $\psi_{0}^{\prime}$. As is well known $[16,17]$, the equilibrium magnetic field stabilizes the interchange modes and, in our case $B_{0}=1$, this is clearly stable. We can therefore expect that in the initial phase the growth of the magnetic island is weakly influenced by interchange parameters. 


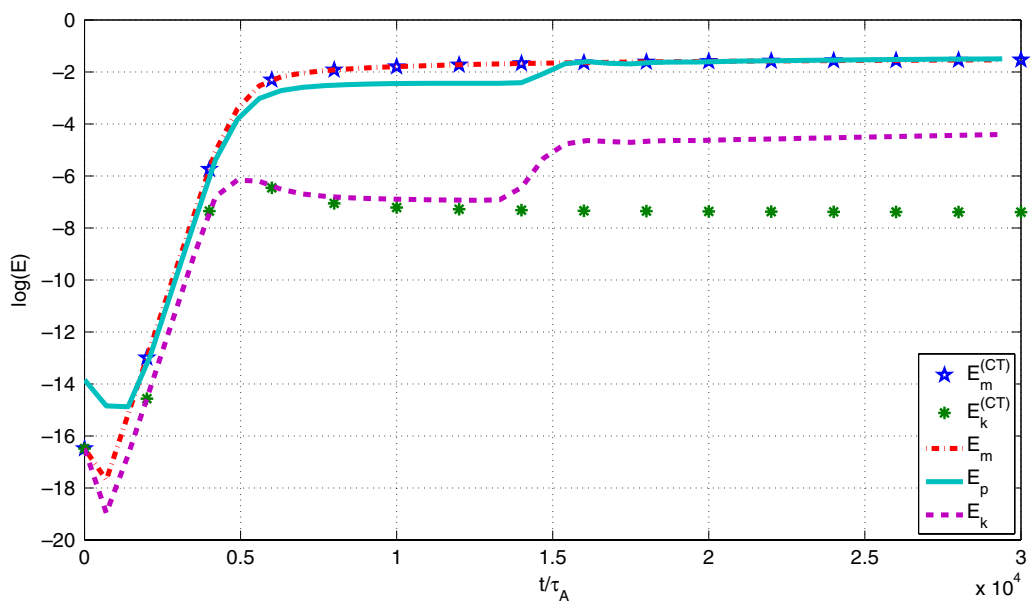

Figure 3. Time evolution of the energies for $\beta=10^{-3}$.
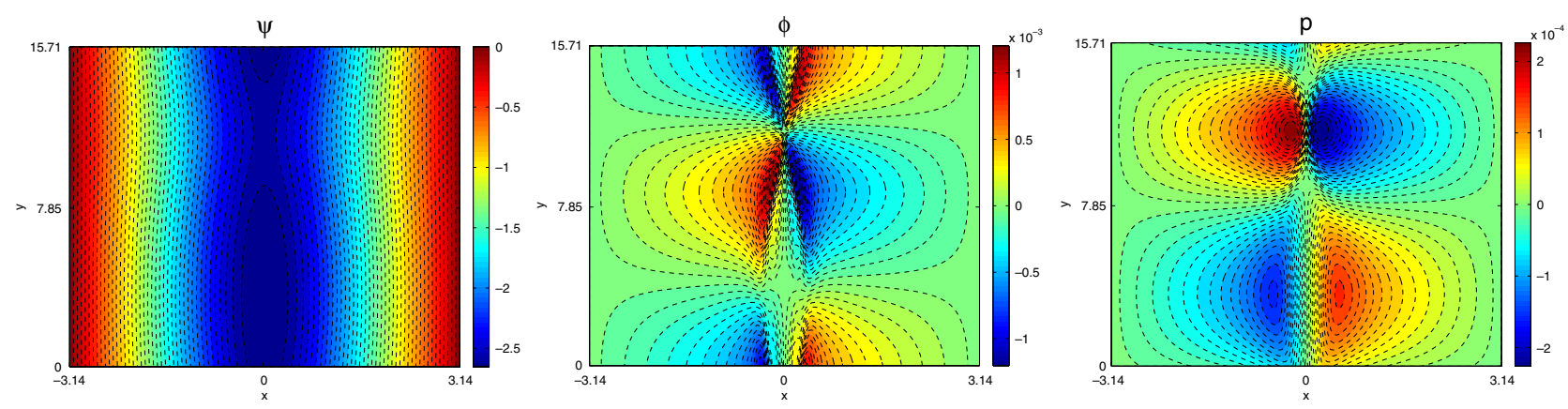

Figure 4. Snapshots of the fields $\psi, \phi$ and $p$ before transition for $\beta=0.001$, at $t=5000 \tau_{\mathrm{A}}$.

\section{Nonlinear generation of a strong zonal flow}

\subsection{Description of the nonlinear evolution of the system}

To characterize how the pressure gradient affects the evolution of a magnetic island, linear and nonlinear self-consistent numerical simulations have been performed. A grid number of $n_{x}=128$ is chosen for the radial direction and $n_{y}=128$ for the poloidal direction (equivalent to 48 modes in this direction, including dealiasing). The energy conservation relation derived from equations (1)-(3) is

$$
\frac{\mathrm{d}}{\mathrm{d} t}\left(E_{\mathrm{m}}+E_{\mathrm{p}}+E_{\mathrm{k}}\right)=-\eta\left\langle j^{2}\right\rangle-v\left\langle\Delta^{2} \phi\right\rangle-\frac{\chi_{\perp}}{C^{2}}\left\langle|\nabla p|^{2}\right\rangle+S,
$$

where $E_{\mathrm{m}}=0.5\left\langle\left|\nabla\left(\psi-\psi_{0}\right)\right|^{2}\right\rangle, E_{\mathrm{p}}=0.5\left\langle p^{2}\right\rangle / C^{2}$ and $E_{\mathrm{k}}=0.5\left\langle|\nabla \phi|^{2}\right\rangle \mid$ are, respectively, the magnetic energy, the pressure energy and the kinetic energy of the fluctuations. The brackets $\langle$.$\rangle mean here an average over the simulation$ domain. $S$ is the source term linked to the curvature and the pressure gradient, proportional to the radial pressure flux, $S=-\alpha_{S}\left\langle p \partial_{y} \phi\right\rangle$ with $\alpha_{S}=\frac{v^{\star}}{C^{2}}\left(1-\kappa_{2}\right)+\kappa_{1}>0$ because $\kappa_{2}<1$. Note that a local flattening of the pressure by radial exchange of pressure cells, gives a fluctuation $\delta S<0$. Moreover, the interchange source term $S$ is not modified by the generation of zonal flow. Figure 3 shows the time evolution of $E_{\mathrm{m}}, E_{\mathrm{p}}$ and $E_{\mathrm{k}}$ for the parameters chosen in this work with $\beta=10^{-3}$ as well as the corresponding $E_{\mathrm{m}}^{\mathrm{CT}}$ and $E_{\mathrm{k}}^{\mathrm{CT}}$, for a classical tearing mode (i.e. $p=0$ and $\kappa_{\mathrm{i}}=0$ ). In comparison with the evolution of a classical tearing mode, four regimes are observed in the nonlinear simulations of a magnetic island in the presence of the interchange term. First, there is a linear regime where the magnetic island is formed. Second, the system reaches a quasi-plateau phase. Then, a transition occurs and as will be shown later, this is linked to the interchange parameters. Finally, the system reaches a new saturated state. During the first two phases, the evolution of the energies is not strongly affected by the presence of the curvature terms. The evolution of the magnetic island closely follows the time trajectory of an island driven by a tearing instability. However, at $t_{\star}=13200 \tau_{\mathrm{A}}$, a transition occurs. Figures 4 and 5 show snapshots of the fields $\psi, \phi$ and $p$, respectively, before and after the transition. The two dimensional profiles of the pressure and the electrostatic potential (represented through isocontours) are strongly affected by this transition. After this phase, the structure of the mode changes and a flattening of the pressure is obtained.

\subsection{Origin of the transition}

In order to understand the origin of the transition and to characterize the structure of the electrostatic potential after the transition, we first assess the importance of small scales. The spectra before the transition $(a)$ and during the transition (b) are shown in figure 6. An equipartition between the energy of the magnetic flux and the energy of the pressure is observed at large scales $0.8<k_{y}<5$, whereas there is an equipartition 

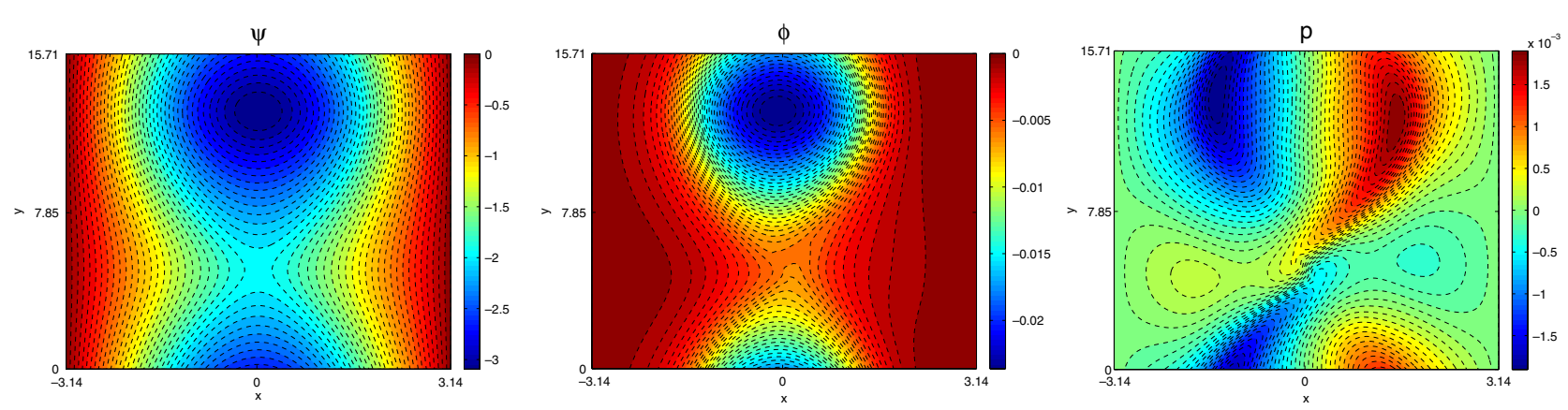

Figure 5. Snapshots of the fields $\psi, \phi$ and $p$ after transition for $\beta=0.001$, at $t=18000 \tau_{\mathrm{A}}$.
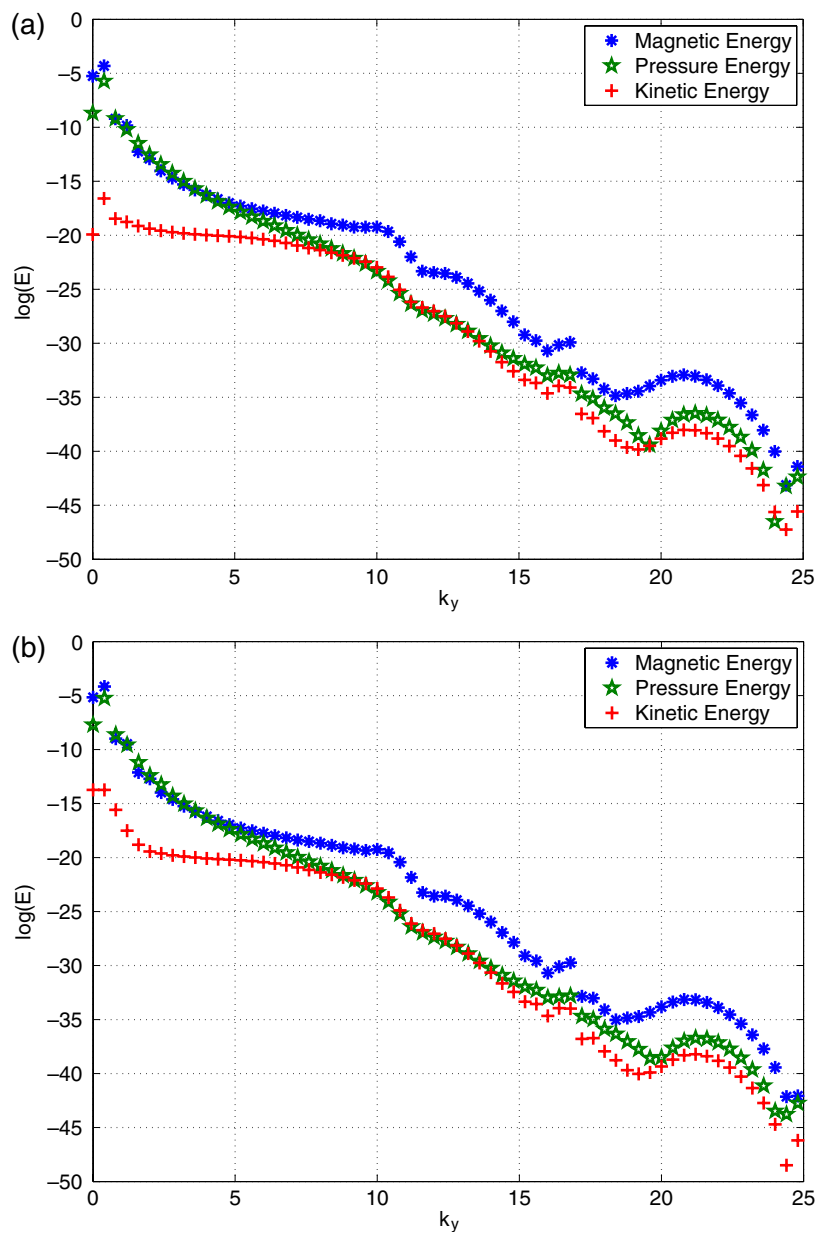

Figure 6. Spectra before $(a)$ and during the transition $(b)$, at respectively $t=12000 \tau_{\mathrm{A}}$ and $t=14500 \tau_{\mathrm{A}}$.

between the energy of the pressure and the kinetic energy at small scales $8>k_{y}>14$. We observe that these properties continue to persist when the transition occurs, the energy level of the modes $k_{y}>1.2$ being roughly unaltered. We also note that as apparent in figure 6 only the large scales are affected at the transition. A detailed analysis shows that the transition occurs when the kinetic energy of the mode $k_{y}=0$ becomes equal to the one of $k_{y}=0.4$ (mode 1 ). This suggests that the dynamics of the structure of the electrostatic potential is quasilinear and that the transition occurs when the mode $k_{y}=0$ becomes energetically dominant. To delineate the quasilinear nature of the magnetic island dynamics, we have performed a simulation with only four poloidal modes and with the same parameters $\left(\beta=10^{-3}\right)$. Figure 7 shows the time evolution of the energies for this simulation. The comparison with figure 3 demonstrates that one needs only four modes to describe the time evolution of the energies. Therefore the dynamics of the system is the result of quasilinear effects. Further, the time evolutions of the kinetic energies of modes 0,1 and 2 are presented in the right hand side panel of figure 7 and compared with the evolution of modes in a classical tearing run, i.e. without pressure effects $\left(p=0\right.$ and $\left.\kappa_{i}=0\right)$. In the latter case, the growth is driven by mode 1 , the transition does not occur and mode 0 is not generated. Conversely, in the case where the pressure effects are included, the transition occurs and the mode $k_{y}=0$, i.e. zonal flow, is strongly generated. It is also the first to be amplified exponentially, at the beginning of the transition. This suggests that the transition is linked to a strong amplification of the zonal flow. Nevertheless, when we perform the same run, suppressing artificially mode 0 , i.e. the zonal flow, we find that the transition still occurs, roughly at the same time, but with a weaker amplitude. This suggests that although zonal flows play a predominant role they are not the sole factor responsible for the destabilizing mechanism. Indeed we can show from the analysis of the snapshots of $\phi, p$ and $\psi$ given by figure 8 that mode 0 plays an important role in the triggering mechanism of the transition phase. We can observe that during the transition, the pressure cells are crossing the resonant surface at the current sheet in both directions, producing at the end a modification of the mode structure. Clearly the potential structure suggests that the crossing results from an advection by the flow. Let us stress that during this phase, the classical tearing picture of an incoming flow from the sheet into the island is no more valid. Between $t=15000 \tau_{\mathrm{A}}$ and $t=17000 \tau_{\mathrm{A}}, S(t)$ decreases because the reorganization of the cell is radially equivalent to an exchange of pressure cells with the gradient of the pressure fluctuations being outward. From an energetic point of view, see equation (9), $\kappa_{1}$ and $\kappa_{2}$ have a negligible effect because the dominant contribution in the interchange source term is linked to $\omega_{\star} / C^{2}$. In figure 7 , the results of a simulation with four modes and without the curvature terms are presented $\left(\kappa_{1}=\kappa_{2}=0\right)$. We find that the transition does not occur. At least, it does not occur at $2.5 t_{\star}$, showing that a more complex mechanism due to curvature terms might be at play. We next investigate the origin of the zonal flow. 

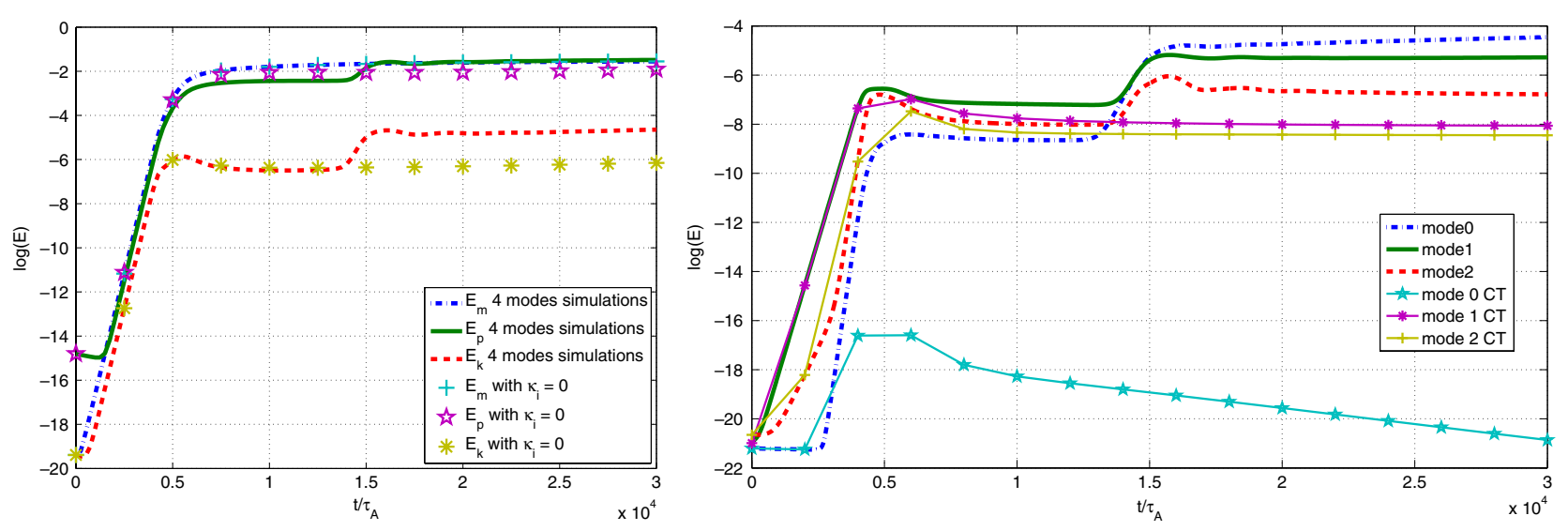

Figure 7. Runs with same parameters as figure 3: (Left) Time evolution of the energies with $\kappa_{1}=\kappa_{2}=0$ (4 modes runs). (Right) Time evolution of the kinetic energy of modes 0,1 and 2 along with the corresponding classical tearing case.
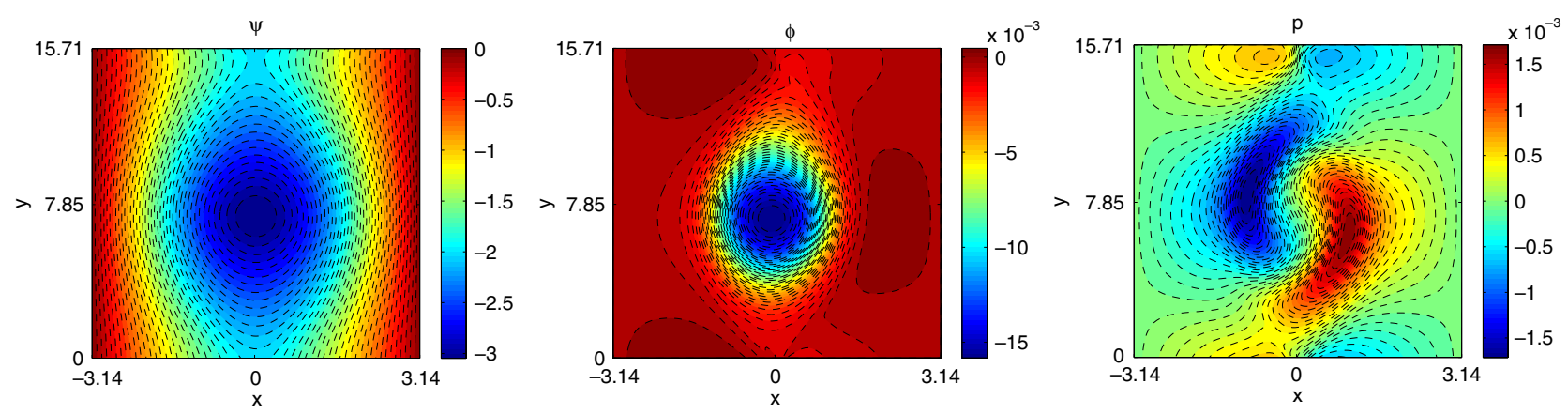

Figure 8. Snapshots of the fields $\psi, \phi$ and $p$ for $\beta=0.001$, at $t=15000 \tau_{\mathrm{A}}$.

\subsection{Origin of the strong increase in the zonal flow}

We investigate the origin of the zonal flow that is generated whenever $\beta \neq 0$ by considering separately the energy transfer from the tearing mode to the zonal flow and also from the other modes, in particular, the small scales.

Following [8], the equation for the flow energy feeding the mode $k_{m}=m 2 \pi / L_{y}$ can be written as

$$
\frac{\mathrm{d}}{\mathrm{d} t} E_{m}=T_{m}^{\mathrm{R}}+T_{m}^{\mathrm{M}}+T_{m}^{\mathrm{C}}+T_{m}^{\mathrm{LB}}+T_{m}^{\mathrm{KI}}
$$

where

$$
\begin{aligned}
T_{m}^{\mathrm{R}} & =-\int \mathrm{d} x \phi_{m}([\phi, \omega])_{m} \text { (Reynolds stress contribution), } \\
T_{m}^{\mathrm{M}} & =\int \mathrm{d} x \phi_{m}([\psi, j])_{m}(\text { Maxwell stress contribution }), \\
T_{m}^{\mathrm{C}} & =-\kappa_{1} k_{m} \int \mathrm{d} x \phi_{m} p_{m} \text { (curvature term contribution), } \\
T_{m}^{\mathrm{LB}} & =\int \mathrm{d} x \phi_{m}\left(\left[\psi_{0}, j\right]\right)_{m} \text { (line bending term contribution), } \\
T_{m}^{\mathrm{KI}} & =\int \mathrm{d} x \phi_{m}\left(\left[\psi, j_{0}\right]\right)_{m} \text { (kink term contribution). }
\end{aligned}
$$

Here $\phi_{m}=\hat{\phi}_{m} \mathrm{e}^{\mathrm{i} k y}+\hat{\phi}_{-m} \mathrm{e}^{-\mathrm{i} k y}$, where $\hat{\phi}_{m}$ is the $m$ Fourier component of $\phi$.

In order to understand the origin of the generation of the zonal flow, it is useful to project equation (10) on the

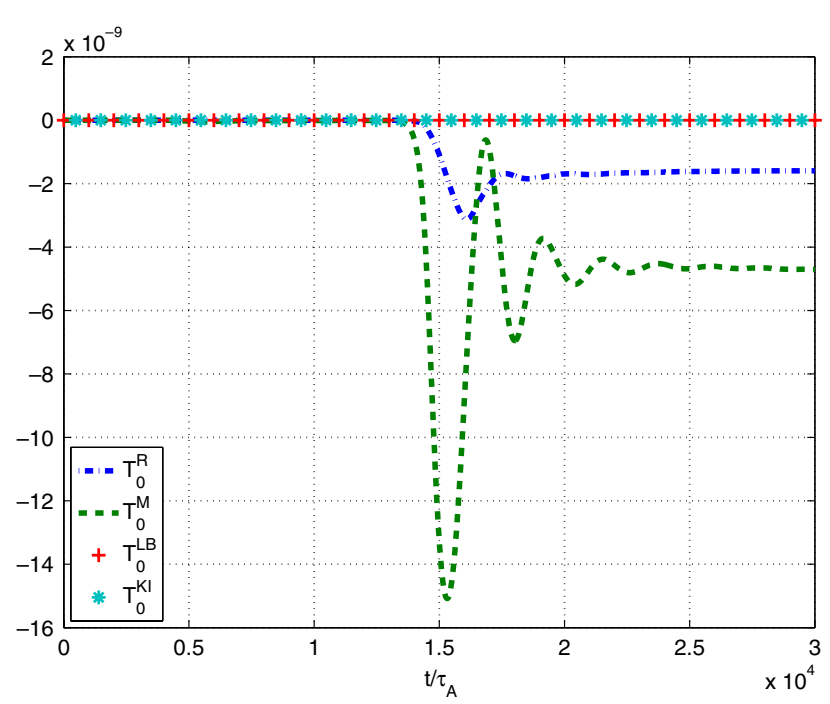

Figure 9. $\beta=10^{-3}$ : Time evolution of $T_{0}^{\mathrm{R}}, T_{0}^{\mathrm{M}}, T_{0}^{\mathrm{LB}}$ and $T_{0}^{\mathrm{KI}}$.

mode $m=0$ :

$$
\frac{\mathrm{d}}{\mathrm{d} t} E_{0}=T_{0}^{\mathrm{R}}+T_{0}^{\mathrm{M}}+T_{0}^{\mathrm{LB}}+T_{0}^{\mathrm{KI}}
$$

The curvature term does not directly feed the zonal flow. In figure 9, the time evolutions of $T_{0}^{\mathrm{R}}, T_{0}^{\mathrm{M}}, T_{0}^{\mathrm{LB}}$ and $T_{0}^{\mathrm{KI}}$ for the simulation with $\beta=10^{-3}$ are presented. The contributions of the line bending and the kink terms are very weak. However, at the transition, there is a strong generation of the Reynolds stress 

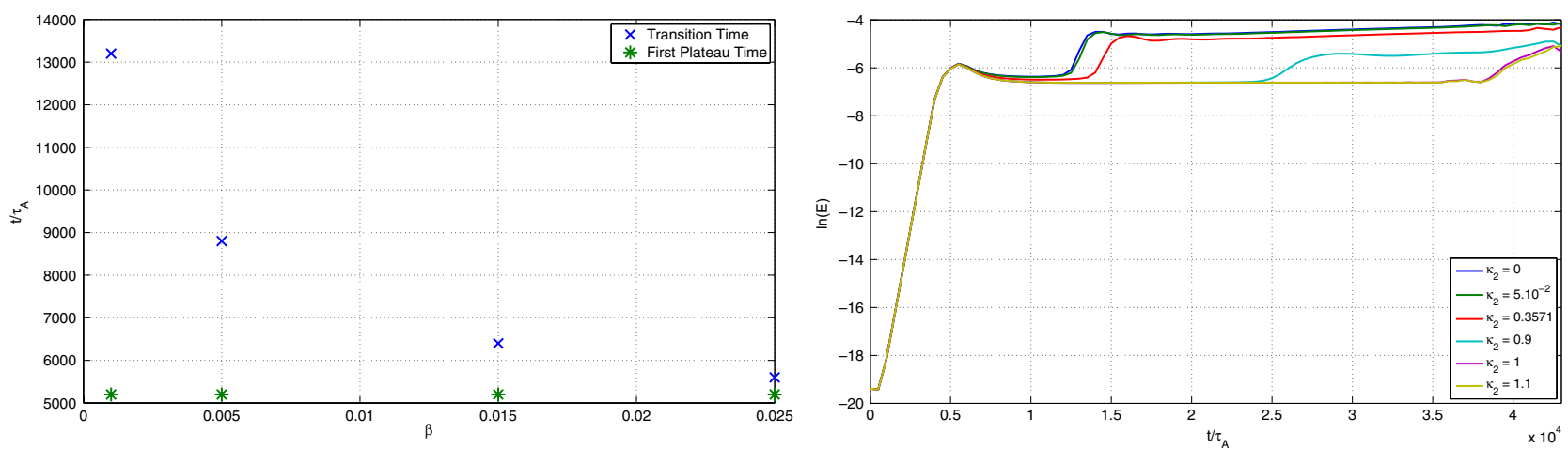

Figure 10. Effect of the $\beta$ parameter and of $\kappa_{2}$ on the transition: (Left) Time at the beginning of the first quasi-plateau phase and time at the beginning of the transition versus $\beta$. (Right) Time evolution of the kinetic energy for simulations with $\beta=10^{-3}$ but with different values of $\kappa_{2}$.

and the Maxwell stress contributions. To proceed further, it is useful to separate the contributions from zonal flow $(m=0)$, the mode $m=1$ and other modes for each of the transfer functions. Let us introduce $\phi_{>1}=\sum_{m>1} \phi_{m}$. We can define three contributions in each transfer function. For instance, in the case of $T_{m}^{\mathrm{R}}$, we have

$$
T_{m}^{\mathrm{R}}=T_{0 m}^{\mathrm{R}}+T_{1 m}^{\mathrm{R}}+T_{>1 m}^{\mathrm{R}},
$$

where $T_{0 m}^{\mathrm{R}}=\int \mathrm{d} x \phi_{m}\left(\left[\phi_{0}, \omega_{0}\right]\right)_{m}, T_{1 m}^{\mathrm{R}}=\int \mathrm{d} x \phi_{m}\left(\left[\phi_{1}, \omega_{1}\right]\right)_{m}$, and $T_{>1 m}^{\mathrm{R}}=\int \mathrm{d} x \phi_{m}\left(\left[\phi_{>1}, \omega_{>1}\right]\right)_{m}$. Clearly, by definition $T_{11}^{\mathrm{R}}=0$ and $T_{0 m}^{\mathrm{R}}=T_{0 m}^{\mathrm{M}}=0$. Let us focus on the energy transfer to zonal flow, neglecting the weak contributions of the line bending and the kink terms. Equation (11) then becomes

$$
\frac{\mathrm{d}}{\mathrm{d} t} E_{0}=T_{10}^{\mathrm{R}}+T_{>10}^{\mathrm{R}}+T_{10}^{\mathrm{M}}+T_{>10}^{\mathrm{M}}
$$

Using the above prescription we have checked that the main contribution to the Reynolds and Maxwell stresses comes from the mode $m=1$ while the contributions of the small scales are weak.

\subsection{Effect of the $\beta$ parameter on the nonlinear dynamics}

The left panel of figure 10 presents the transition time and the time where the first quasi-plateau saturation occurs for various values of $\beta$. We note that the time corresponding to the first quasi-plateau phase does not depend on the $\beta$ parameter. This is in agreement with the results of figure 3 , which shows the time evolution of the energies for two cases, namely the classical tearing case $(\beta=0)$ and the $\beta=10^{-3}$ case. However the saturation time depends strongly on the $\beta$ parameter. For a regime where the pressure effects are strong, i.e. for a high value of $\beta$, the transition occurs quickly whereas, for low $\beta$ regimes, the transition occurs later. Further, in the right panel of figure 10, the effect of the interchange parameter $\kappa_{2}$ on the transition is shown for a simulation with four modes and with $\beta=10^{-3}$. The transition time clearly depends on the $\kappa_{2}$ parameter. It tends to stabilize the first plateau phase.

To summarize, the nonlinear transition results from quasilinear effects. Zonal flow amplification at the transition is due to the energy transfer from the mode $m=1$ to the mode $m=0$ mainly through the Maxwell stress. The curvature term $\kappa_{2}$ linked to the interchange effect does not directly feed the growth of the zonal flow. However, we have shown that this term controls the transition time $t_{\star}$. The transition leads to an effective radial exchange of pressure cells generating an outward mean pressure gradient of fluctuations. The shape of the pressure structure after the transition implies that a diamagnetic velocity $\tilde{\omega}_{\star}$ has been nonlinearly generated, driving a rotation of the island. This driving is of course in competition with the zonal flow. Let us in the next section analyse quantitatively the island poloidal rotation.

\section{Study of the island poloidal rotation}

\subsection{Model for the island rotation frequency}

Following [4] where a study of the rotation frequency of the island has been done for the case of a drift tearing mode, we investigate the origin of the magnetic island poloidal rotation. Let us project Ohm's law (equation (3)) on the mode $m=1$ assuming that for mode $k_{y}, \tilde{\psi}_{k_{y}}(x, y, t)=\psi_{k_{y}}(x) \mathrm{e}^{\mathrm{i} k_{y} y} \mathrm{e}^{-\mathrm{i} \gamma t}$, where the real part of $\gamma$ is the frequency of the island rotation and the imaginary part is the linear growth rate of the island. Neglecting the nonlinear contribution of the modes $k_{y}>k_{1}$, we obtain the expression for the rotation frequency $\omega$ of the island

$$
\omega=\omega^{\star}+\tilde{\omega}^{\star}+\tilde{\omega}_{E \times B}+L_{\psi_{0}}+L_{\eta}
$$

where

$$
\begin{aligned}
& \omega^{\star}=k_{1} v^{\star}, \\
& \tilde{\omega}^{\star}=-k_{1} \partial_{x} p_{0}, \\
& \tilde{\omega}_{E \times B}=k_{1} \partial_{x} \phi_{0}, \\
& L_{\psi_{0}}=-\operatorname{Re}\left(k_{1} \psi_{0}^{\prime} \frac{\phi_{k_{1}}(x)-p_{k_{1}}(x)}{\psi_{k_{1}}(x)}\right), \\
& L_{\eta}=\operatorname{Re}\left(i \eta \frac{\left(\partial_{x}^{2}-k_{1}^{2}\right) \psi_{k_{1}}(x)}{\psi_{k_{1}}(x)}\right),
\end{aligned}
$$

are respectivly the linear diamagnetic drift, the nonlinear diamagnetic drift, the contribution of the equilibrium magnetic field and the contribution of the resistivity. In general, each term of equation (14) is not a constant inside the current sheet $\delta$, 


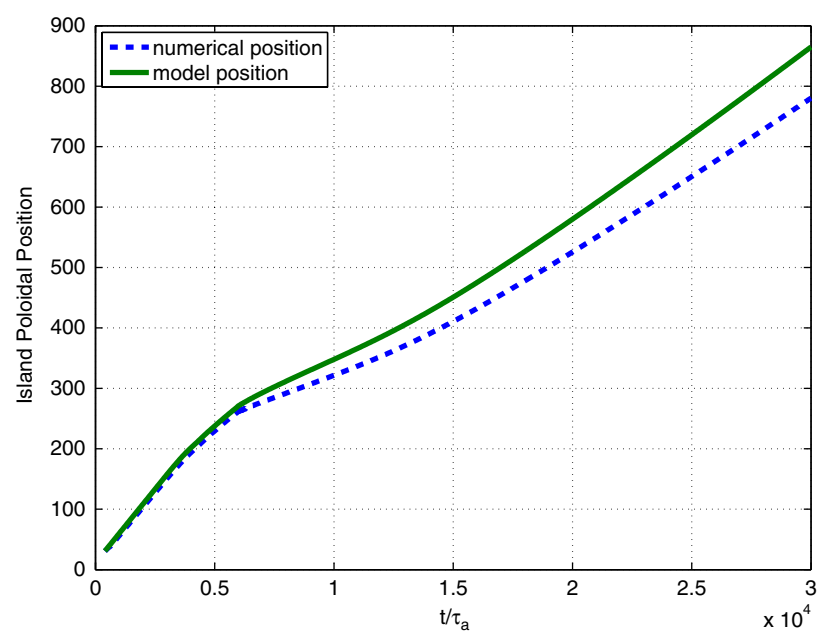

Figure 11. Time evolution of the poloidal position of the island: comparison between the model and numerical data for $\beta=0.025$.

so we consider their radial averages over the current sheet to contribute to the rotation frequency. Equation (14) becomes:

$$
\langle\omega\rangle_{\delta}=\left\langle\omega^{\star}+\tilde{\omega}^{\star}\right\rangle_{\delta}+\left\langle\tilde{\omega}_{E \times B}\right\rangle_{\delta}+\left\langle L_{\psi_{0}}\right\rangle_{\delta}+\left\langle L_{\eta}\right\rangle_{\delta},
$$

where $\langle.\rangle_{\delta}$ means an average over the current sheet.

In figure 11, the time evolution of the poloidal position is presented for a nonlinear simulation with $\beta=0.025$. The dynamics of the energies for a simulation with $\beta=0.025$ has the same behaviour as the one obtained in figure 3 with $\beta=10^{-3}$. However, as shown in the left panel of figure 10 , with such a high value of $\beta$, the transition occurs earlier around $t=5600 \tau_{\mathrm{A}}$. In figure 11 a comparison with the island position obtained from the model equation (15) is also shown. The derived model is in agreement with the numerical data and the dynamics of the island rotation is recovered. We would like to mention here that the time integration for these results has been performed on a very long time scale compared with the Alfvén time.

Equation (15) shows that the effective island frequency is the result of different contributions. In order to investigate the effect of the interchange terms on the island rotation, figure 12 presents the evolution of each frequency for a simulation with $\beta=0.025$ setting $\kappa_{1}=\kappa_{2}=0$ (left panel) and for a simulation with $\beta=0.025$ including the curvature/interchange terms (right panel). First, for the two simulations, the contributions to the rotation of the equilibrium magnetic field $L_{\psi_{0}}$ and of the resistivity $L_{\eta}$ are weak. Moreover, figure 12 shows that the frequency dynamics is not affected by the curvature terms during the linear regime and during the first quasiplateau phase. Actually, during the linear formation of the magnetic island, the rotation is controlled mainly by the linear diamagnetic drift while $\tilde{\omega}^{\star}$ and $\tilde{\omega}_{E \times B}$ are weak. During the first quasi-plateau phase, the nonlinear diamagnetic drift and the $E \times B$ poloidal flow are strongly generated, and affect the island rotation. The $E \times B$ poloidal flow is the most important contribution to the frequency during this regime. However, after the first quasi-plateau phase, interchange terms affect the dynamics of the frequency. When the interchange terms are switched off, the time evolution of the frequencies is in agreement with previous results found for a drift tearing mode [4]. After the nonlinear generation of the flows, linear and nonlinear diamagnetic drifts cancel each other. As a result, the $E \times B$ poloidal flow controls the effective frequency of the island, $\omega_{t} \sim \tilde{\omega}_{E \times B}$. The right panel of figure 12 shows that when interchange terms are included, such cancelling of the total diamagnetic frequency does not occur anymore after the transition. Hence the total diamagnetic drift then provides the main contribution to the island rotation. However, after the transition, clearly $\partial \omega_{t} / \partial t \sim \partial \omega_{E \times B} / \partial t$. The asymptotic island velocity is enhanced by the curvature terms $\kappa_{1}$ and $\kappa_{2}$.

\subsection{Origin of the $E \times B$ flow}

Nonlinearly, the $E \times B$ flow is generated and affects the rotation of the island. In order to investigate its origin, the flow equation (equation (1)) is projected on the mode $k_{1}$ for the limiting case of $\nabla_{\perp}^{2} \approx \partial_{x}^{2}$. We obtain

$$
\begin{aligned}
\partial_{t} \partial_{x}^{2} \phi_{0}= & -\frac{1}{L_{y}} \int_{L_{y}}\left[\phi, \nabla_{\perp}^{2} \phi\right] \mathrm{d} y+\frac{1}{L_{y}} \int_{L_{y}}\left[\psi, \nabla_{\perp}^{2} \psi\right] \mathrm{d} y \\
& +\frac{\mu}{L_{y}} \int_{L_{y}} \partial_{x}^{4} \phi \mathrm{d} y
\end{aligned}
$$

We have defined the $E \times B$ poloidal flow as $\tilde{\omega}_{E \times B}=$ $k_{1} \partial_{x} \phi_{0}$. So multiplying equation (16) by $k_{1}$ and averaging over the current sheet $\delta$, we obtain

$$
\partial_{t}\left\langle\tilde{\omega}_{E \times B}\right\rangle_{\delta}=R(t)+M(t)+V(t),
$$

where

$$
\begin{aligned}
& R(t)=-\frac{k_{1}}{\delta L_{y}} \int_{\delta} \int_{L_{y}}\left[\phi, \nabla_{\perp}^{2} \phi\right] \mathrm{d} y \mathrm{~d} x, \\
& M(t)=\frac{k_{1}}{\delta L_{y}} \int_{\delta} \int_{L_{y}}\left[\psi, \nabla_{\perp}^{2} \psi\right] \mathrm{d} y \mathrm{~d} x, \\
& V(t)=\frac{\mu k_{1}}{\delta L_{y}} \int_{\delta} \int_{L_{y}} \partial_{x}^{4} \phi \mathrm{d} y \mathrm{~d} x
\end{aligned}
$$

with $R(t)$ being the Reynolds stress, $M(t)$ being the Maxwell stress and $V(t)$ being the viscosity contribution to the $E \times B$ flow.

On the left panel of the figure 13, the time evolutions of $R, M$ and $V$ are presented for a simulation with $\beta=0.025$. As expected, the stresses are nonlinearly generated at the beginning of the first quasi-plateau phase allowing the growth of the $E \times B$ poloidal flow. Except at the end of the linear regime where the Reynolds stress is not yet generated, the viscosity term is very weak and does not play an important role in the generation of the flow. The most important contributions come from the Reynolds and Maxwell stresses which balance each other. There is a strong amplification of the amplitude of the stresses during the transition. On the right panel of figure 13, a closeup of the temporal dynamics of $R, M$ and $V$ during the transition time evolutions are presented. Note that during this transition, whereas the viscosity term is still weak, the evolutions of the Reynolds and Maxwell stresses are complementary. The two stresses tend to balance each other during this phase where the flow is crossing the separatrices, limiting the level of the generated zonal flow, even if they are both growing in amplitude. Once the transition 

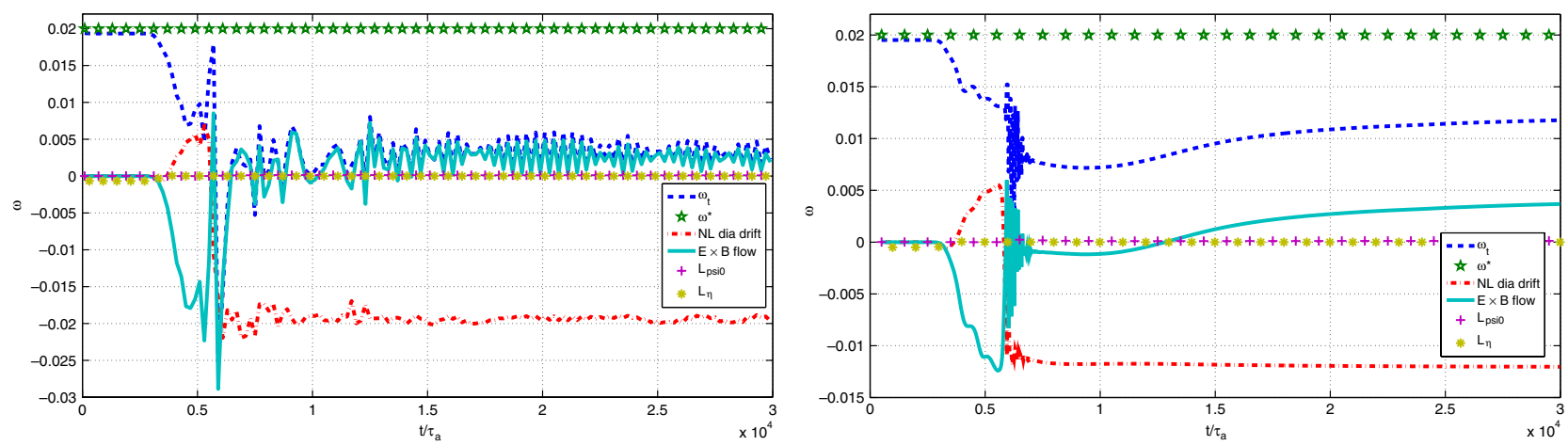

Figure 12. Time evolution of the frequencies for simulations with $\beta=0.025$ : (Left) Simulation setting $\kappa_{1}=\kappa_{2}=0$. (Right) Simulation including the interchange terms.
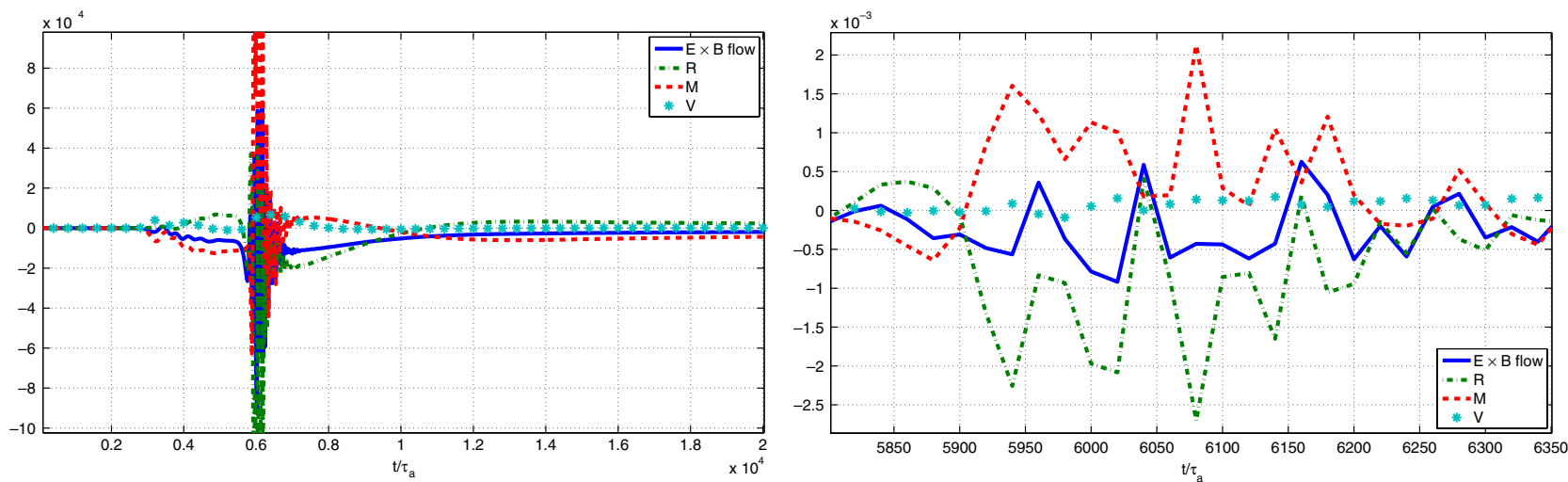

Figure 13. Time evolution of $\mathrm{M}, \mathrm{R}$ and $\mathrm{V}$ for $\beta=0.025$ : (Left) Evolution from $t=0 \tau_{\mathrm{A}}$ to $t=30000 \tau_{\mathrm{A}}$ of the stresses over the current sheet. (Right) Zoom during the transition of the stresses over the current sheet.

has occurred, the amplitude of the mean nonlinear brackets in the vicinity of the separatrix, $R$ and $M$, fall. However, in the new dynamical equilibrium the resulting $E \times B$ poloidal flow persists asymptotically and is driven by the Maxwell stress, as a response of the magnetic structure to the new field distribution.

\subsection{Origin of the nonlinear diamagnetic drift}

In order to investigate the origin of the nonlinear diamagnetic drift, we follow the same procedure for the pressure equation (equation (2)). After projection on mode $k_{1}$, we obtain:

$$
\partial_{t}\left\langle\tilde{\omega}^{\star}\right\rangle_{\delta}=\mathrm{d} C(t)+\mathrm{d} M(t)+D(t)
$$

where

$$
\begin{array}{r}
\mathrm{d} C(t)=-\frac{k_{1}}{\delta L_{y}} \int_{\delta} \int_{L_{y}} \partial_{x}[\phi, p] \mathrm{d} y \mathrm{~d} x, \\
\mathrm{~d} M(t)=\frac{C^{2} k_{1}}{\delta L_{y}} \int_{\delta} \int_{L_{y}} \partial_{x}\left[\psi, \nabla_{\perp}^{2} \psi\right] \mathrm{d} y \mathrm{~d} x, \\
D(t)=\frac{k_{1} \chi_{\perp}}{\delta L_{y}} \int_{\delta} \int_{L_{y}} \partial_{x}^{3} p \mathrm{~d} y \mathrm{~d} x .
\end{array}
$$

$\mathrm{d} C$ is the contribution to the nonlinear diamagnetic drift of the divergence of the convective term, $\mathrm{d} M$ is the contribution of the divergence of the Maxwell stress and $D$ is the contribution of the diffusivity.

In the left panel of figure 14, the time evolutions of $\mathrm{d} C$, $\mathrm{d} M$ and $D$ are presented. The three contributions to the nonlinear diamagnetic drift are generated at the beginning of the nonlinear regime. During the first quasi-plateau phase, $\mathrm{d} C$, $\mathrm{d} M$ and $D$ participate actively in the generation and growth of the nonlinear diamagnetic drift $\tilde{\omega}^{\star}$. At the transition there is an amplification of the amplitude of the three contributions. The right panel of figure 14 presents a closeup of the time evolutions of $\mathrm{d} C, \mathrm{~d} M$ and $D$ during the transition. During this transition, the contribution of the diffusivity does not grow and is relatively weak compared with $\mathrm{d} C$ and $\mathrm{d} M$. Note that both the divergence of the convective term and of the Maxwell stress feed the nonlinear diamagnetic drift. We observe that in the first phase of the transition, the nonlinear diamagnetic drift is driven by $\mathrm{d} C$, the term linked to the advection of the pressure cells. In the following phase this term is balanced by the Maxwell divergence stress leading to a stabilization of the island dynamics. This shows the importance of the coupling parameter $C$ during the transition. In the saturation phase, the origin of $\tilde{\omega}^{\star}$ comes mainly from the divergence of the convective term, $\mathrm{d} M$ and $D$ becoming relatively weak. 

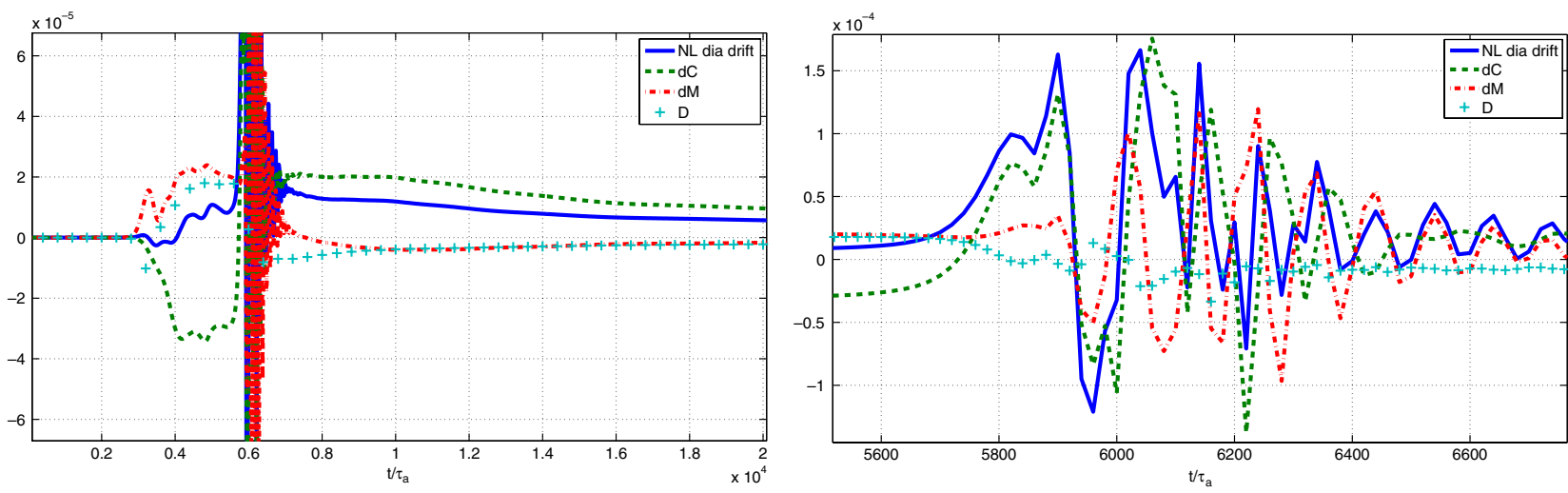

Figure 14. Time evolution of $\mathrm{d} C, \mathrm{~d} M$ and $D$ for $\beta=0.025$ : (Left) Evolution from $t=0 \tau_{\mathrm{A}}$ to $t=30000 \tau_{\mathrm{A}}$ over the current sheet. (Right) Zoom during the transition over the current sheet.

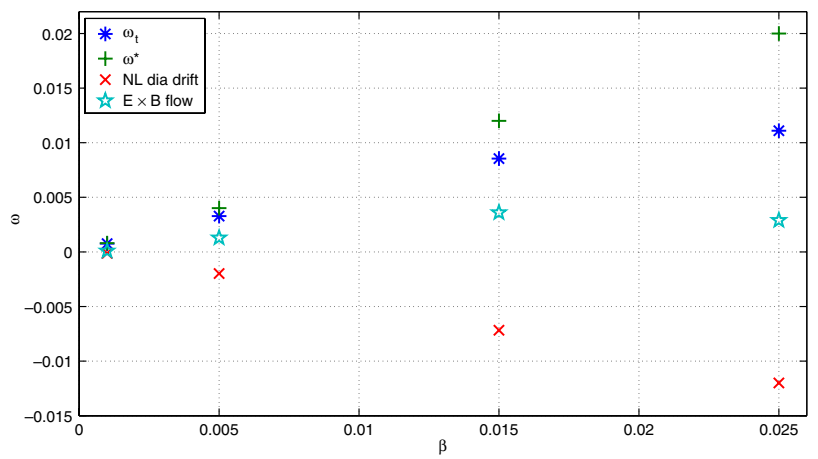

Figure 15. Effect of $\beta$ on the rotation frequency after the transition.

\subsection{Effect of the $\beta$ parameter on the island poloidal rotation}

In figure 15 , the effect of the $\beta$ parameter on the rotation frequency is presented. The frequencies have been time averaged from $t=15000 \tau_{\mathrm{A}}$ to $t=30000 \tau_{\mathrm{A}}$. At low $\beta$, pressure effects are weak and the situation is close to the classical tearing situation. The competition between Reynolds and Maxwell stresses produces nonlinearly neither the zonal flow nor a diamagnetic drift. The asymptotic rotation frequency $\omega_{t}$ increases with $\beta$. The nonlinear diamagnetic frequency increases almost linearly with $\beta$, but with a slope lower than the linear one, allowing a global asymptotic drift of the island in the electron diamagnetic direction. Let us note, however, that the direction of the island rotation depends on the value of the viscosity parameter [4]. For any $\beta$, the zonal flow contribution to the island drift is weaker than the diamagnetic one. Note also that from $\beta \sim 0.015, \omega_{E \times B}$ decreases, and this is linked to the transition observed in figure 16 where the effect of the $\beta$ parameter on the average stresses is shown (from $t=15000 \tau_{\mathrm{A}}$ to $\left.t=30000 \tau_{\mathrm{A}}\right)$. The amplification of the stresses leads to the nonlinear generation of the mean flows and hence considerably affects the rotation of the magnetic island. More precisely, it appears that for low $\beta$ values, the amplitudes of the stresses are very weak as in the classical tearing case. This explains why nonlinear diamagnetic drift and nonlinear $E \times B$ poloidal flow do not strongly affect the rotation of the island in those cases. However, for high $\beta$ regimes, the amplitude of the stresses, in particular the Maxwell and convective contribution become more important.
At $\beta \sim 0.015$, a transition is observed: first, $\mathrm{d} M$ ceases to be negligible compared with $\mathrm{d} C$, second the Reynolds contribution $R$ starts to grow, weakening the global $E \times B$ flow, as observed in figure 15 .

\section{Summary}

The nonlinear dynamics of a magnetic island in the presence of pressure gradient effects has been investigated. This nonlinear dynamics is different from the classical tearing case and exhibits a bifurcation. After a linear growth of the island and a first quasi-plateau phase, a transition occurs and the system reaches a new saturated state characterized by the flattening of the pressure profile. We have shown that the dynamics of the island during this bifurcation is due to quasilinear effects. The strong generation of a zonal flow, due to interchange terms, allows this transition to occur. We have shown that the time at which the transition occurs decreases with $\beta$ while it increases with the pressure parameter $\kappa_{2}$. Regarding the poloidal rotation of the magnetic island, a model including quasilinear effects has been tested successfully. Before the transition, the rotation of the island corresponds to the linear diamagnetic drift. Then, at the transition, the rotation is strongly affected by the nonlinear generation of the diamagnetic drift and of the $E \times B$ flow. We have shown that the asymptotic nonlinear diamagnetic drift is a linear function of $\beta$ but does not cancel the linear drift, as previously obtained when curvature parameters are neglected. The diamagnetic effect appears to be the dominant contribution to the island rotation. We have also shown that the $\beta$ parameter affects the magnetic rotation through an amplification of the stresses. We have provided a detailed analysis of their impacts on the $E \times B$ and diamagnetic drifts for both the transition and the asymptotic regime. At high $\beta$, we find that a Reynolds stress is generated in the vicinity of the island and weakens the influence of the asymptotic $E \times B$ flow on the rotation.

\section{Acknowledgments}

The authors wish to thank S. Nishimura, D. Escande and G. Fuhr for fruitful discussions. M.M. acknowledges the College Doctoral Franco-Japonais for its fellowship. This work is partly supported by LIA 336 CNRS and by NIFS/NINS 

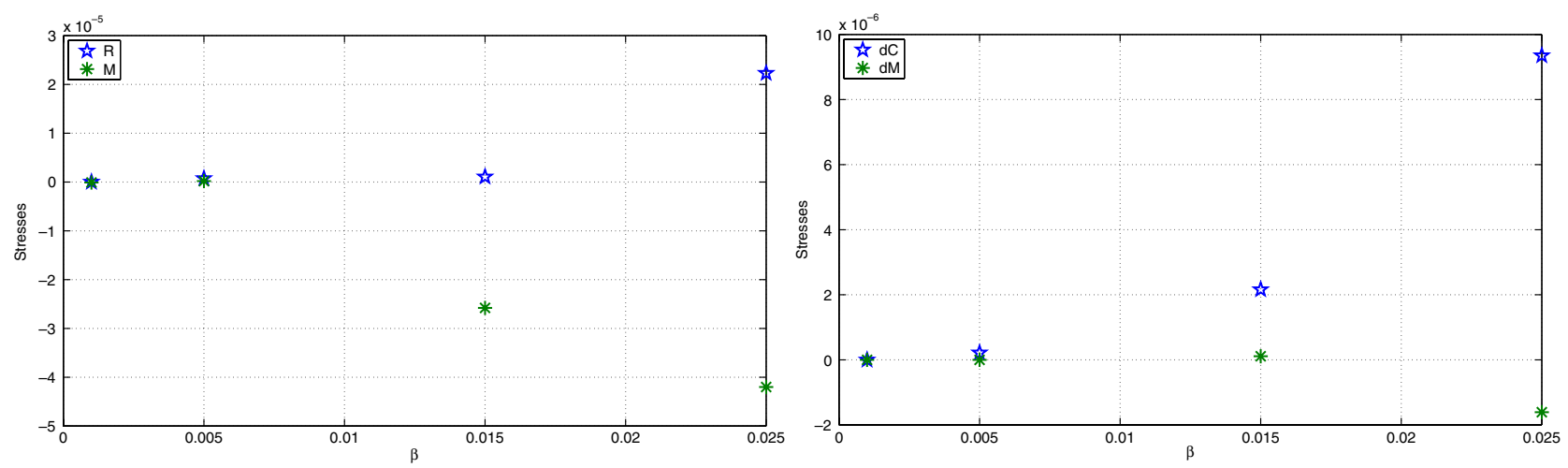

Figure 16. Effect of $\beta$ on the stresses after the transition: (Left) Reynolds and Maxwell stresses versus $\beta$ parameter. (Right) Divergence of the convection and divergence of the Maxwell stress versus $\beta$.

under the project of Formation of International Network for Scientific Collaborations. This work has been supported by a grant from Agence Nationale de la Recherche ANR-05BLAN-0183-03.

\section{References}

[1] Priest E.R. 1981 Solar Flare Magnetohydrodynamics (New York: Gordon and Breach)

[2] Horton W., Kim J.-H., Militello F. and Ottaviani M. 2007 Phys. Plasmas 14012902

[3] Ottaviani M., Porcelli F. and Grasso D. 2004 Phys. Rev. Lett. 93075001

[4] Nishimura S., Benkadda S., Yagi M., Itoh S.-I. and Itoh K. 2008 Phys. Plasmas 15092506

[5] Itoh K., Itoh S.-I., Diamond P.H. and Hahm T.S. 2006 Phys. Plasmas 13055502
[6] Tanaka K. et al 2006 Nucl. Fusion 46110

[7] Takaji S. et al 2002 Nucl. Fusion 42634

[8] Ishizawa A. and Nakajima N. 2007 Nucl. Fusion 471540

[9] Militello F., Waelbroeck F.L., Fitzpatrick R. and Horton W. 2008 Phys. Plasmas 15050701

[10] Hazeltine R.D., Kotschenreuther M. and Morrison P.J. 1985 Phys. Fluids 282466

[11] Arakawa A. 1997 J. Comput. Phys. 135 103-14

[12] Furuya A., Itoh S. and Yagi M. 2001 J. Phys. Soc. Japan 711261

[13] Harris E.G. 1962 Nuovo Cimento 23115

[14] Porcelli F. 1987 Phys. Fluids 301734

[15] Grasso D., Ottaviani M. and Porcelli F. 2001 Phys. Plasmas 84306

[16] Biskamp D. 2000 Magnetic Reconnection in Plasmas (Cambridge: Cambridge University Press)

[17] Biskamp D. 1993 Nonlinear Magnetohydrodynamics (Cambridge: Cambridge University Press) 